\title{
Modulation by $\beta$-Aminopropionitrile of Vessel Luminal Narrowing and Structural Abnormalities in Arterial Wall Collagen in a Rabbit Model of Conventional Balloon Angioplasty versus Laser Balloon Angioplasty
}

\author{
J. Richard Spears, ${ }^{*}$ Hong Zhan, ${ }^{*}$ Sandeep Khurana, ${ }^{*}$ Robert L. Karvonen, ${ }^{*}$ and Karen M. Reiser* \\ *Department of Medicine, Division of Cardiology, Harper Hospital/Wayne State University School of Medicine, Detroit, Michigan \\ 48201; and ${ }^{\ddagger}$ Department of Medicine, University of California, Davis, California 95616
}

\begin{abstract}
This study was designed to assess the potential relationship between the late loss of angiographic luminal diameter and biochemical abnormalities of arterial wall collagen in rabbits subjected to angioplasty, and to test the hypothesis that $\beta$-aminopropionitrile ( $\beta \mathrm{APN})$, an inhibitor of lysyl oxidase, would inhibit such changes when administered orally for 1 mo after angioplasty. Endovascular injury was induced in rabbit iliac arteries by ipsilateral balloon angioplasty (BA) and by contralateral balloon angioplasty accompanied by exposure to continuous wave neodymium: yttrium aluminum garnet laser radiation (LBA). Computer measurement of angiographic luminal diameter demonstrated significant vessel narrowing at 1 and 6 mo after both procedures. By quantitative histology, the majority of the 1-mo loss in angiographic diameter could not be attributed to neointimal thickening. Analysis of collagen cross-linking by HPLC in collagen obtained from the LBA-injured segments of the arteries 1 mo after angioplasty revealed a significant increase, relative to values from uninjured arteries $(P$ $<0.05)$, in the difunctional cross-link dihydroxylysinonorleucine (DHLNL). 6 mo after angioplasty, the content of hydroxpyridinium, the trifunctional maturational product of DHLNL, was significantly elevated in both BA- and LBA-treated arteries compared with values from uninjured arteries $(P<0.05)$. In animals administered $\beta$ APN, luminal narrowing at $1 \mathrm{mo}$, compared with controls, was attenuated $(P<0.01)$ and DHLNL content was decreased $(P<0.05)$ in arteries subjected to LBA, but not in arteries subjected to $B A$. The results suggest that lathyrogenic agents may be efficacious in favorably modulating LBA-induced alterations in vessel diameter and mural connective tissue. (J. Clin. Invest. 1984. 93:1543-1553.) Key words: angioplasty $\bullet$ collagen $\bullet \beta$-aminopropionitrile $\bullet$ lysyl oxidase inhibitor $\bullet$ laser
\end{abstract}

\section{Introduction}

The pathogenesis of lesion recurrence after initially successful percutaneous transluminal coronary angioplasty and alternative angioplasty procedures is not well defined, but neointimal cellular proliferation and thrombus formation have been im-

Address correspondence to Dr. Karen M. Reiser, California Primate Research Center, County Road \#98, Hutchinson Drive, Davis, CA 95616.

Received for publication 26 March 1993 and in revised form 30 September 1993.

J. Clin. Invest.

(C) The American Society for Clinical Investigation, Inc.

$0021-9738 / 94 / 04 / 1543 / 11 \$ 2.00$

Volume 93, April 1994, 1543-1553 plicated as components of new tissue growth (1-7). In addition, production of extracellular matrix, principally collagen and glycosaminoglycans, within the neointima has been shown to be a prominent feature of the late phase of restenosis lesions, both experimentally and clinically (8-10). A less well-recognized hypothesis regarding late luminal compromise is that collagen synthesis may also contribute to intramural fibrosis and possibly to cicatricial contracture after angioplasty injury to all three layers of the arterial wall. In the classic wound-healing response observed in other tissues, an increase in the collagen cross-link dihydroxylysinonorleucine (DHLNL) ${ }^{1}$ per mole of collagen accompanies biosynthesis of collagen (11) and may contribute to stricture formation. However, to our knowledge, no studies have been reported regarding collagen crosslinking as a potential contributory mechanism in the chronic loss in luminal dimensions after angioplasty.

The lathyrogen, $\beta$-aminopropionitrile ( $\beta \mathrm{APN})$, is an irreversible inhibitor of lysyl oxidase (12), an extracellular enzyme that promotes crosslink formation in nascent fibrils of both collagen and elastin by conversion of lysine and hydroxylysine side chain residues into aldehydes. Only growing, i.e., newly synthesized collagen fibrils, are rendered lathyrogenic by $\beta A P N$ (13), and its administration experimentally and clinically as an antifibrotic agent reduces the mechanical strengh of collagen and may enhance collagenolysis (14-17). As a result, scar contracture is prevented by $\beta A P N$ in conditions that would ordinarily be characterized by prominent cross-linking of newly synthesized collagen.

This study was designed to investigate the potential relationship between angioplasty-induced late vessel luminal narrowing and biochemical alterations in arterial wall collagen, and to determine the feasibility of modulating such changes by administration of $\beta$ APN. The specific hypotheses tested were: (a) that the extracellular matrix that accumulates in the arterial wall after angioplasty is structurally abnormal, with cross-linking patterns characteristic of other injury/repair processes known to culminate in deposition of excess collagen; and (b) that inhibition of abnormal cross-linking of collagen by administration of $\beta \mathrm{APN}$ would reduce the magnitude of late luminal compromise after angioplasty injury in a rabbit model.

\section{Methods}

\section{Animals}

New Zealand White rabbits, weighing 3.5-4.2 kg, were obtained from Harlan Sprague-Dawley (Indianapolis, IN) and subsequently housed and studied in a facility approved by the American Association for the

1. Abbreviations used in this paper: BA, balloon angioplasty; $\beta \mathrm{APN}$, $\beta$-aminopropionitrile; DHLNL, dihydroxylysinonorleucine; LBA, laser balloon angioplasty. 
Accreditation of Laboratory Animal Care. In a protocol approved by the Wayne State University Animal Investigation Committee, all animals received humane care in compliance with the "Principles of Laboratory Animal Care" formulated by the National Society for Medical Research and the "Guide for the Care and Use of Laboratory Animals" prepared by the National Academy of Sciences (NIH Publication 8523 , revised 1985). All rabbits were maintained on standard rabbit chow (Ralston Purina) ad libitum throughout the course of the study.

Rabbits were assigned either to a treatment group $(n=12)$, which received $\beta$ APN fumarate (Sigma) $300 \mathrm{mg} / \mathrm{kg}$ orally (dissolved in drinking water) for 1 month after angioplasty or to a control group $(n=18)$, which did not receive the drug after angioplasty. In addition, five 3.5$4.2-\mathrm{kg}$ rabbits and five 4.2-kg retired breeders, not subjected to angioplasty, served as controls for arterial wall collagen analysis. The quantity of water consumed was examined daily for each rabbit in the treatment group to ensure that the target dose of $\beta$ APN was administered.

\section{Angioplasty procedure}

Angioplasty was performed bilaterally on the external iliac artery in a manner similar to that described previously (18). Briefly, a 5-French sheath was advanced into the thoracic aorta via a carotid arteriotomy performed under sterile technique and ketamine and xylazine anesthesia. Either balloon angioplasty (BA) or laser balloon angioplasty (LBA) was randomly performed in the right or left iliac artery with the same balloon catheter (USCI Spears ${ }^{\mathrm{TM}}$ LBA catheter, USCI Div., C. R. Bard, Inc., Billerica, MA). The contralateral iliac artery was then subjected to the alternative angioplasty modality, so that both types of angioplasty procedures were always performed in each rabbit. The balloon size, either 3.0 or $3.5 \mathrm{~mm}$, was selected to increase luminal diameter during inflation by $>10 \%$ compared with baseline diameter, and the same balloon was used for both BA and LBA in each rabbit. An inflation pressure of 4 bar was used for both procedures, as was a 1-min period of balloon occlusion. For LBA, a 20-s continuous wave neodymium: yttrium aluminum garnet (cw Nd: YAG) laser exposure $(1.06 \mu \mathrm{m})$ of 375 and $475 \mathrm{~J}$ for the 3.0- and 3.5-mm balloons, respectively, was delivered from a $17-\mathrm{mm}$-long cylindrical diffusing tip during balloon inflation, in a manner similar to that used clinically. A peak tissue temperature of $90-100^{\circ} \mathrm{C}$ has been shown experimentally to be achieved during such laser exposures (19). Upon completion of the procedure, the rabbits were allowed to recover from anesthesia. All rabbits received heparin $100 \mathrm{U} / \mathrm{kg}$ i.v. immediately before to arterial entry and antibiotic prophylaxis with Wycillin $150,000 \mathrm{U}$ i.m. (Wyeth). No further medications, except $\beta A P N$ for rabbits in the treatment group, were given after angioplasty.

\section{Serial angiography}

Aortography was performed with $8 \mathrm{~cm}^{3}$ Omnipaque injected at $2 \mathrm{~cm}^{3} / \mathrm{s}$ through polyethylene 90 tubing positioned $1 \mathrm{~cm}$ above the iliac bifurcation immediately before and after the interventional procedures in each rabbit. 1 mo after angioplasty, aortography was repeated in the same manner in all animals. Although the primary goal of the study was to evaluate angiographic luminal dimensions and collagen crosslinking over a 1-mo period of time, which corresponds to a "late" follow-up as defined herein, some of the rabbits treated were allowed to recover at this time, and a final aortogram was obtained 6 mo after angioplasty. $\beta$ APN was discontinued for those rabbits treated with the drug during the first month. For the 1- and 6-mo follow-up procedures, the aortogram was performed both before and after intraaortic injection of nitroglycerin $50 \mu \mathrm{g}$ in each rabbit in order to assess the potential effect of vasoconstriction on luminal diameter.

Angiographic images were recorded at $50 \%$ optical magnification for all studies by use of a zoom lens mounted on a video camera at the output phosphor of a fluoroscopic unit (Precise Optics, Bayshore, NY) with a 6-in image intensifier and recorded on a 3/4-in Sony videorecorder. Video frames were digitized in a $640 \times 480$ array, 8-bit gray scale format at an anatomic resolution of $\sim 10$ pixels $/ \mathrm{mm}$ with a frame grabber interfaced to a MicroVax II computer (Digital Equipment Corp., Maynard, MA), and automated luminal edge tracking was used to determine mean vessel diameter over a $1-\mathrm{cm}$ axial length of the arterial segment corresponding to the midportion of the 2-cm-long balloon. Computer analysis of the inflated balloon, a noncompliant one (polyethylene terephthalate) having a well-defined diameter at 4 bar (either 3.0 or $3.5 \mathrm{~mm}$ ) used for the angioplasty procedure, was performed to obtain absolute vessel luminal diameter. The accuracy of this extensively validated automated angiographic luminal edge tracking algorithm, developed by us (20-22), has been shown to be within $100 \mu \mathrm{m}$ of actual anatomic luminal diameter over a broad range of radiographic conditions.

\section{Quantitative histology}

Although the 1-mo mean loss in luminal diameter after either BA or LBA of the external iliac artery of the normocholesterolemic NZW rabbit has been shown (18) to be similar in magnitude to that noted after clinical angioplasty of coronary arteries, the magnitude of neointimal thickening has not previously been quantitated for this animal model. It should be noted that, in this current study, hypercholesterolemia was not induced primarily for two reasons. Serum cholesterol levels achieved experimentally on a hypercholesterolemic diet typically far exceed those found clinically; in addition, the response in serum cholesterol is heterogeneous, which may increase the variability in the tissue responses to injury, thereby potentially obscuring differences between treatment strategies.

To determine the contribution of neointimal thickening to luminal compromise $1 \mathrm{mo}$ after angioplasty in the normocholesterolemic rabbit model, 10 control rabbits, each of which had been subjected to BA and LBA, were killed at this time for quantitative histologic analysis. The segments of external iliac arteries corresponding to the site of angioplasty were removed under fluoroscopic control immediately after a final aortogram and placed in $10 \%$ neutral buffered formalin. The segments were not perfused fixed in order to confirm a previously noted effect of laser/thermal exposure on straightening of the elastic lamellae (18), since a ruffled appearance of the lamellae normally is enhanced without laser/thermal exposure in sections not perfused fixed. After immersion in graded alcohol solutions and embedment in paraffin, microtome sections obtained from the midportion of each specimen were stained with hematoxylin-eosin (H \& E), Masson's trichrome, and elastin stains. High quality sections, either H \& E- or Masson's trichrome-stained ones, were viewed at $\times 75$ with a video camera mounted on a microscope (Reichert-Jung, Buffalo, NY), and video images were digitized by a frame grabber interfaced to a MicroVax II computer. Neointimal and medial areas were automatically determined after manual delineation of the internal and external elastic lamellae and the luminal boundary.

Because of marked retraction and recoil that occurs upon postmortem removal of an arterial section in the rabbit, the histologic luminal area is artifactually much smaller than that in vivo. Therefore, to estimate the contribution of neointimal thickening to the angiographic diameter loss over $1 \mathrm{mo}$, the luminal area was extrapolated from the angiographic diameter measurement with the assumption of circular symmetry. Neointimal thickness was then estimated as follows:

$\pi\left(\left[D_{\mathrm{N}} / 2\right]^{2}\right)=\pi\left(\left[D_{\mathrm{L}} / 2\right]^{2}\right)+A_{\mathrm{H}}$,

where $D_{\mathrm{N}}=$ diameter of the region bounded by the internal elastic lamella, $\pi\left(\left[D_{\mathrm{N}} / 2\right]^{2}\right)=$ luminal area plus neointimal area, $D_{\mathrm{L}}=$ angiographic luminal diameter, and $A_{\mathrm{H}}=$ histologic neointimal area. Solving for $D_{\mathrm{N}}$,

$D_{\mathrm{N}}=\left(\left[D_{\mathrm{L}}\right]^{2}+4 A_{\mathrm{H}} / \pi\right)^{1 / 2}$.

Neointimal thickness, defined as $\left(\left[D_{\mathrm{N}}-D_{\mathrm{L}}\right] / 2\right)$, then was derived:

$\left[D_{\mathrm{N}}-D_{\mathrm{L}}\right] / 2=\left\{\left(\left[D_{\mathrm{L}}\right]^{2}+4 A_{\mathrm{H}} / \pi\right)^{1 / 2}-D_{\mathrm{L}}\right\} / 2$.

Medial thickness was derived from histologic measurement of medial area, neointimal area, and angiographic luminal diameter in the same manner. 


\section{Biochemical analyses}

Immediately after death at either 1 or $6 \mathrm{mo}$, iliac artery segments subjected to angioplasty were removed, rinsed in normal saline, and frozen at $-70^{\circ} \mathrm{C}$ until analysis. Additional control rabbits, including five 3.5$\mathrm{kg}$ rabbits and five $5.0-\mathrm{kg}$ retired breeders (representing two different age groups), none of which had been subjected to angioplasty, were killed for similar analysis.

DHLNL and HLNL. DHLNL and HLNL, the major difunctional lysyl oxidase-mediated cross-links in arterial collagen, were reductively labeled as follows: specimens of artery weighing $25-50 \mathrm{mg}$ (wet weight) were minced into fine pieces, washed in neutral salt buffer, and reduced with $\mathrm{NaB}_{3} \mathrm{H}_{4}$ (142 Ci/Mol; Amersham Corp., Arlington Heights, IL) as described by us in detail previously $(23,24)$. The effective reducing capacity of each batch of $\mathrm{NaB}_{3} \mathrm{H}_{4}$ was determined using $\delta$-amino levulinic acid as a standard compound for reduction, thus allowing us to determine molar quantities of cross-links from their radioactivity (24). Reduced tissues were hydrolyzed in $6 \mathrm{~N} \mathrm{HCl}$ and hydroxyproline content was determined colorimetrically (25). Reduced DHLNL and HLNL were analyzed by reverse-phase HPLC under isocratic conditions, using a sodium phosphate-buffered mobile phase containing $n$ propanol and SDS, as described in detail previously (24). The collagen content of the samples was calculated from the hydroxyproline content. Fractions were collected at 1 -min intervals for liquid scintillation counting. Data are expressed as moles of crosslink per mole of collagen.

Hydroxypyridium (OHP). The mature, nonreducible crosslink OHP was quantified by reverse-phase HPLC; the mobile phase consisted of $22 \%$ acetonitrile in distilled water with heptafluorobutyic acid as an ion-pairing agent as described in detail by us previously (24). Cross-links were detected fluorometrically with a fluorometer (2000; Hitachi, Tarrytown, NY) equipped with a xenon bulb (excitation = $295 \mathrm{~nm}$; emission $=395 \mathrm{~nm}$ ). Preparation and calibration of standards has been described in detail previously. Data are expressed as moles of crosslink per mole of collagen.

\section{Statistical analysis}

For comparisons of changes in mean angiographic luminal diameter over 1 mo between treatment groups (1, control [no $\beta A P N] / B A ; 2$, control [no $\beta$ APN]/LBA; $3, \beta$ APN/BA; and $4, \beta$ APN/LBA) at each time period, a two-way analysis of variance (ANOVA) was used. Differences between groups were considered significant for $P$ values $<0.05$, after adjustment for multiple comparisons with a Bonferroni-type correction. ANOVA was also used for comparisons of DHLNL, HLNL, and OHP between groups. For comparison of mean luminal diameters of the same vessel segments over time as well as before and after nitroglycerin, a paired Student's $t$ test was used. Results are expressed as mean \pm 1 SD.

\section{Results}

\section{Angiographic luminal diameter}

Conventional BA. (Figs. 1-3; Tables I and II) The mean acute increase in luminal diameter was similar $(P>0.05)$ in the control (no $\beta$ APN) and $\beta$ APN-treated groups ( 15 and $20 \%$, respectively). The drug had no apparent effect on the chronic luminal diameter response to the procedure, in that the mean 1-mo loss in luminal diameter, compared with the acute postangioplasty result, was nearly identical (25\%) for both groups.

$L B A$. (Figs. 1-3; Tables II and IV) Acutely, the mean increase in luminal diameter for both the control and $\beta A P N$ treated groups (21 and 19\%, respectively) was similar ( $P$ $>0.05$ ) between these two groups and compared to both BA groups. In the control group, i.e., LBA without $\beta A P N$, the 1mo mean loss in luminal diameter (23\%), compared with the acute postangioplasty result, was similar $(P>0.05)$ to that found for the BA groups. However, rabbits treated with $\beta A P N$ demonstrated only a $10 \%$ mean loss in luminal diameter $1 \mathrm{mo}$

Table I. BA without $\beta A P N$

\begin{tabular}{|c|c|c|c|c|c|c|c|}
\hline Rabbit & Balloon size & Pre-BA & Post-BA & $1 \mathrm{mof} / \mathrm{u}$ & $6 \mathrm{mo} \mathrm{f} / \mathrm{u}$ & $\begin{array}{l}\text { Percent change } \\
\text { (1 mo-pre)/pre }\end{array}$ & $\begin{array}{l}\text { Percent change } \\
\text { (1 mo-post)/post }\end{array}$ \\
\hline & $m m$ & $m m$ & $m m$ & $m m$ & $m m$ & & \\
\hline CB 1 & 3.0 & 2.52 & 2.99 & 1.71 & & -32.14 & -42.81 \\
\hline CB 2 & 3.0 & 2.40 & 2.42 & 1.88 & 2.12 & -21.67 & -22.31 \\
\hline CB 3 & 3.0 & 2.38 & 2.69 & 2.26 & 1.90 & -5.04 & -15.99 \\
\hline CB 4 & 3.0 & 2.58 & 2.82 & 2.51 & 2.36 & -2.71 & -10.99 \\
\hline CB 5 & 3.0 & 2.14 & 2.72 & 1.37 & 1.61 & -35.98 & -49.63 \\
\hline CB 6 & 3.0 & 2.10 & 3.08 & 1.60 & & -23.81 & -48.05 \\
\hline CB 7 & 3.0 & 2.10 & 2.52 & 1.87 & & -10.95 & -25.79 \\
\hline CB 8 & 3.0 & 2.35 & 3.15 & 2.42 & & 2.98 & -23.18 \\
\hline CB 9 & 3.0 & 2.23 & 2.77 & 2.13 & & -4.48 & -23.11 \\
\hline CB 10 & 3.5 & 3.17 & 3.63 & 3.31 & & 4.42 & -8.82 \\
\hline CB 11 & 3.0 & 2.91 & 3.07 & 2.49 & & -14.43 & -18.89 \\
\hline CB 12 & 3.0 & 2.28 & 3.14 & 2.22 & & -2.63 & -29.30 \\
\hline CB 13 & 3.5 & 2.95 & 3.40 & 2.75 & & -6.78 & -19.12 \\
\hline CB 14 & 3.0 & 2.29 & 2.97 & 2.06 & & -10.04 & -30.64 \\
\hline CB 15 & 3.5 & 3.11 & 3.41 & 2.85 & & -8.36 & -16.42 \\
\hline CB 16 & 3.0 & 2.48 & 2.93 & 2.54 & & 2.42 & -13.31 \\
\hline CB 17 & 3.0 & 2.26 & 3.40 & 2.44 & & 7.97 & -28.24 \\
\hline CB 18 & 3.0 & 2.80 & 2.94 & 2.20 & & -21.43 & -25.17 \\
\hline Mean & & 2.50 & 3.00 & 2.26 & 2.00 & -10.15 & -25.10 \\
\hline SD & & 0.34 & 0.32 & 0.47 & 0.32 & 12.54 & 11.76 \\
\hline
\end{tabular}

Luminal diameter by computer analysis of digitized angiograms before (pre), acutely after (post), and at 1 and 6 mo after angioplasty. f/u, follow-up. 


\begin{tabular}{|c|c|c|c|c|c|c|c|}
\hline Rabbit & Balloon size & Pre-LBA & Post-LBA & $1 \mathrm{mof} / \mathrm{u}$ & $6 \mathrm{mof} / \mathrm{u}$ & $\begin{array}{l}\text { Percent change } \\
\text { (1 mo-pre)/pre }\end{array}$ & $\begin{array}{l}\text { Percent change } \\
\text { (1 mo-post)/post }\end{array}$ \\
\hline & $m m$ & $m m$ & $m m$ & $m m$ & $m m$ & & \\
\hline CL 1 & 3.0 & 2.23 & 2.99 & 1.97 & & -11.66 & -34.11 \\
\hline CL 2 & 3.0 & 2.39 & 2.89 & 2.31 & 2.27 & -3.35 & -20.07 \\
\hline CL 3 & 3.0 & 2.32 & 2.91 & 2.19 & 2.43 & -5.60 & -24.74 \\
\hline CL 4 & 3.0 & 2.50 & 2.94 & 2.06 & 2.04 & -17.60 & -29.93 \\
\hline CL 5 & 3.0 & 2.20 & 2.73 & 2.37 & 2.34 & 7.73 & -13.19 \\
\hline CL 6 & 3.0 & 2.48 & 3.10 & 2.32 & & -6.45 & -25.16 \\
\hline CL 7 & 3.0 & 2.49 & 2.77 & 2.23 & & -10.44 & -19.49 \\
\hline CL 8 & 3.0 & 2.36 & 3.15 & 2.47 & & 4.66 & -21.59 \\
\hline CL 9 & 3.0 & 2.21 & 2.78 & 2.02 & & -8.60 & -27.34 \\
\hline CL 10 & 3.5 & 3.13 & 3.45 & 3.25 & & 3.83 & -5.80 \\
\hline CL 11 & 3.0 & 2.81 & 3.08 & 2.36 & & -16.01 & -23.38 \\
\hline CL 12 & 3.0 & 2.46 & 3.12 & 2.29 & & -6.91 & -26.60 \\
\hline CL 13 & 3.5 & 3.07 & 3.50 & 2.86 & & -6.84 & -18.29 \\
\hline CL 14 & 3.0 & 2.51 & 2.56 & 2.07 & & -17.53 & -41.85 \\
\hline CL 15 & 3.5 & 3.02 & 3.48 & 2.35 & & -22.19 & -32.47 \\
\hline CL 16 & 3.0 & 2.43 & 2.88 & 2.46 & & 1.23 & -14.58 \\
\hline CL 17 & 3.0 & 2.57 & 3.20 & 2.71 & & 5.45 & -15.31 \\
\hline CL 18 & 3.0 & 2.80 & 2.94 & 2.11 & & -24.64 & -28.23 \\
\hline Mean & & 2.55 & 3.08 & 2.36 & 2.27 & -7.50 & -23.45 \\
\hline SD & & 0.29 & 0.26 & 0.32 & 0.17 & 9.66 & 8.56 \\
\hline
\end{tabular}

See legend to Table I.

after LBA, which was significantly lower than for the other three groups (two-way ANOVA, $P<0.01$ ). Similarly, compared with the luminal diameter acutely before angioplasty, the diameter at 1 mo was increased by a mean 7\% in LBA-treated arteries in rabbits administered $\beta \mathrm{APN}$, which was significantly different from the $8-13 \%$ mean decrease in this measurement in the other three groups $(P<0.01)$.
6 mo after angioplasty, compared with the 1-mo results, there was no change in the mean luminal diameter for the $\beta A P N$ group for either LBA- or BA-treated arteries, despite discontinuation of the drug at 1 mo after the procedure $(P$ $>0.05$ ). It should be noted that no instance of a late increase in luminal diameter was found (i.e., no evidence of aneurysm formation) in LBA/ $\beta A P N$-treated arteries in any rabbit.

Table III. BA with $\beta A P N$

\begin{tabular}{|c|c|c|c|c|c|c|c|}
\hline Rabbit & Balloon size & Pre-BA & Post-BA & $1 \mathrm{mo} \mathrm{f} / \mathrm{u}$ & $6 \mathrm{mo} \mathrm{f} / \mathrm{u}$ & $\begin{array}{l}\text { Percent change } \\
\text { (1 mo-pre)/pre }\end{array}$ & $\begin{array}{l}\text { Percent change } \\
(1 \text { mo-post)/post }\end{array}$ \\
\hline & $\mathrm{mm}$ & $m m$ & $m m$ & $m m$ & $\mathrm{~mm}$ & & \\
\hline TB 1 & 3.0 & 2.86 & 2.99 & 2.51 & & -12.24 & -16.05 \\
\hline TB 2 & 3.0 & 2.15 & 2.56 & 2.22 & 1.92 & 3.26 & -13.28 \\
\hline TB 3 & 3.5 & 2.95 & 3.41 & 2.68 & 2.79 & -9.15 & -21.41 \\
\hline TB 4 & 3.0 & 1.96 & 2.32 & 1.78 & 1.75 & -9.18 & -23.28 \\
\hline TB 5 & 3.5 & 3.09 & 3.29 & 2.60 & 2.63 & -15.86 & -20.97 \\
\hline TB 6 & 3.0 & 2.36 & 2.58 & 2.05 & & -13.14 & -20.54 \\
\hline TB 7 & 3.0 & 2.45 & 2.87 & 1.91 & 2.05 & -22.04 & -33.45 \\
\hline ТВ 8 & 3.5 & 2.85 & 3.06 & 2.13 & 2.84 & -25.26 & -30.39 \\
\hline TВ 9 & 3.0 & 2.73 & 2.98 & 2.34 & 2.34 & -14.29 & -21.48 \\
\hline TB 10 & 3.5 & 2.33 & 2.96 & 2.13 & 2.32 & -8.58 & -28.04 \\
\hline TB 11 & 3.0 & 2.20 & 2.79 & 2.34 & & 6.36 & -16.13 \\
\hline TB 12 & 3.0 & 2.21 & 2.72 & 1.32 & & -40.27 & -51.47 \\
\hline Mean & & 2.51 & 2.88 & 2.17 & 2.33 & -13.37 & -24.71 \\
\hline SD & & 0.37 & 0.31 & 0.38 & 0.40 & 12.33 & 10.31 \\
\hline
\end{tabular}

See legend to Table I. 


\begin{tabular}{|c|c|c|c|c|c|c|c|}
\hline Rabbit & Balloon size & Pre-LBA & Post-LBA & $1 \mathrm{mof} / \mathrm{u}$ & $6 \mathrm{mo} \mathrm{f} / \mathrm{u}$ & $\begin{array}{l}\text { Percent change } \\
\text { (1 mo-pre)/pre }\end{array}$ & $\begin{array}{l}\text { Percent change } \\
\text { (1 mo-post)/post }\end{array}$ \\
\hline & $m m$ & $m m$ & $m m$ & $m m$ & $m m$ & & \\
\hline TL 1 & 3.0 & 2.49 & 2.94 & 2.35 & & -5.62 & -20.07 \\
\hline TL 2 & 3.0 & 2.13 & 2.73 & 2.49 & 2.46 & 16.90 & -8.79 \\
\hline TL 3 & 3.5 & 3.01 & 3.28 & 2.83 & 3.12 & -5.98 & -13.72 \\
\hline TL 4 & 3.0 & 1.98 & 2.52 & 2.50 & 2.51 & 26.26 & -0.79 \\
\hline TL 5 & 3.5 & 2.76 & 3.26 & 2.93 & 2.98 & 6.16 & -10.12 \\
\hline TL 6 & 3.0 & 2.43 & 2.82 & 2.72 & & 11.93 & -3.55 \\
\hline TL 7 & 3.0 & 2.44 & 2.88 & 2.97 & 2.70 & 21.72 & 3.13 \\
\hline TL 8 & 3.5 & 2.82 & 3.27 & 3.20 & 2.45 & 13.48 & -2.14 \\
\hline TL 9 & 3.0 & 2.31 & 2.89 & 2.43 & 2.45 & 5.19 & -15.92 \\
\hline TL 10 & 3.5 & 2.55 & 2.85 & 2.25 & 2.32 & -11.76 & -21.05 \\
\hline TL 11 & 3.0 & 2.23 & 2.78 & 2.28 & & 2.24 & -17.99 \\
\hline TL 12 & 3.0 & 2.40 & 2.77 & 2.51 & & 4.58 & -9.39 \\
\hline Mean & & 2.46 & 2.92 & 2.62 & 2.62 & $7.10^{*}$ & $-10.03^{*}$ \\
\hline SD & & 0.29 & 0.24 & 0.30 & 0.29 & 11.54 & 7.97 \\
\hline
\end{tabular}

See legend to Table I. ${ }^{*} P<0.01$ compared to BA with $\beta$ APN and to BA or LBA without $\beta$ APN (two-way ANOVA).

Although a slight acute increase (3\%) in luminal diameter was noted after nitroglycerin administration only for BAtreated arteries 1 mo after angioplasty, with or without $\beta \mathrm{APN}$, this change was not statistically significant.

\section{Quantitative histology (Fig. 4)}

Mean neointimal and medial areas of arterial segments from rabbits $(n=10)$ killed 1 mo after angioplasty were similar for BA and LBA $(P>0.05)$. The mean diameter losses at 1 mo for this group of rabbits (15 and $19 \%$ for BA and LBA, respectively) were similar to those for the entire control group ( $P$ $>0.05$ ). When luminal diameter from the angiographic study performed immediately before death at 1 mo was used to extrapolate mean neointimal thickness from the histologic area measurements, mean neointimal thickening was 47 and $20 \mu \mathrm{m}$ for LBA- and BA-treated arteries, respectively. Mean thickness

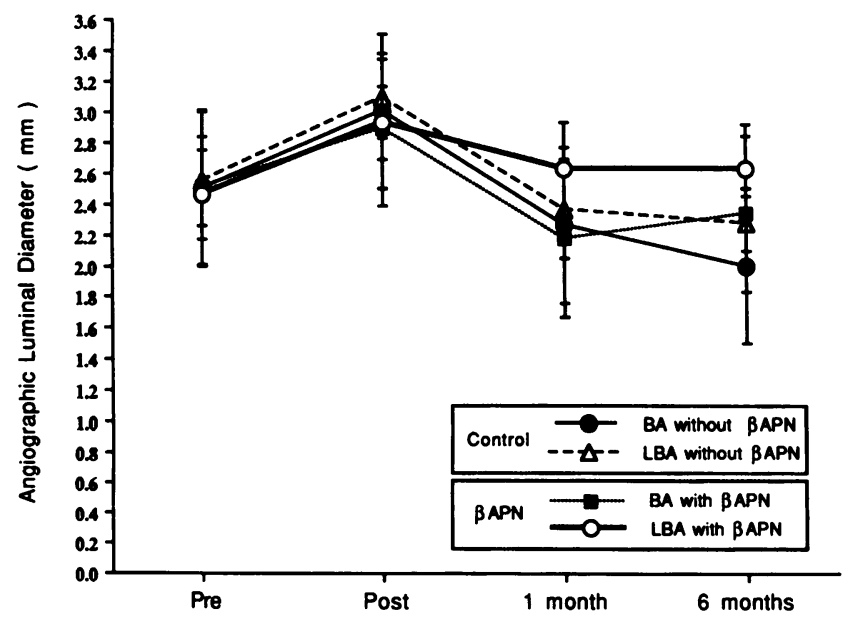

Figure 1. Mean angiographic luminal diameter before (Pre), acutely after (Post), $1 \mathrm{mo}$, and $6 \mathrm{mo}$ after angioplasty (both conventional BA and LBA in each rabbit) of the rabbit external iliac artery, either without $\beta$ APN (control group) or with $\beta$ APN (treatment group). Error bars represent \pm 1 SD. of the media was found by this approach to be 64 and $55 \mu \mathrm{m}$, respectively, for LBA- and BA-treated arteries.

The histologic appearance of the angioplastied arteries was similar between the 1- and 6-mo follow-up periods. The neointima appeared qualitatively similar between BA and LBA at these times, as reported previously (18). However, as also previously reported, by Masson's trichrome stain, LBA-treated arteries demonstrated a prominent network of collagen in the media, compared with BA-treated arteries (Fig. 5). Straightening of the internal and external elastic laminae was apparent in LBA-treated arteries, unlike the ruffled appearance of these structures in BA-treated arteries and arteries not subjected to angioplasty.

\section{Biochemical assays (Tables $V$ and VI)}

DHLNL and HLNL. The reducible cross-links DHLNL and HLNL were readily detectable in arterial collagen. In LBA-

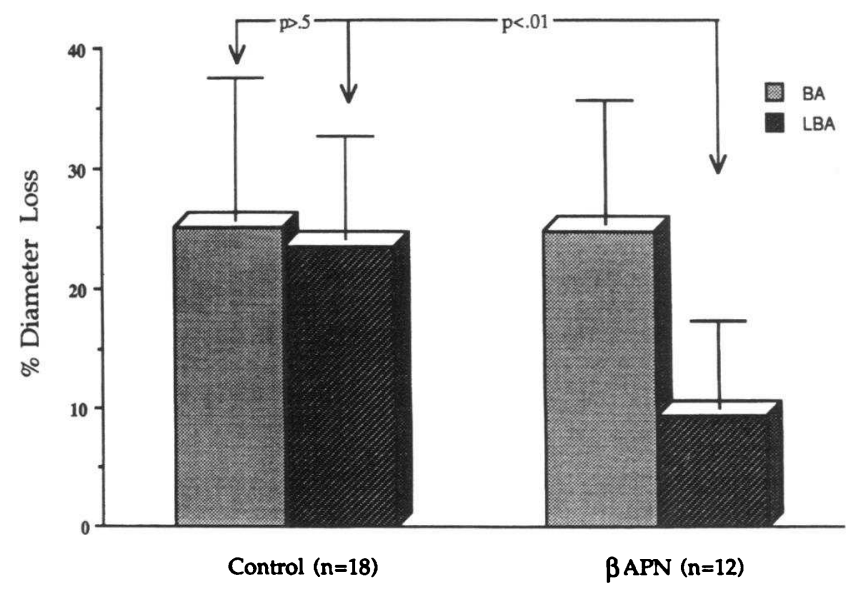

Figure 2. Mean percent loss $( \pm 1 \mathrm{SD})$ in diameter 1 mo after angioplasty (both BA and LBA), either with $\beta$ APN (treatment group) or without $\beta$ APN (control group). The diameter loss for LBA/ $\beta A P N$ treated arteries was significantly less $(P<0.01$ by two-way ANOVA) than that for LBA without $\beta \mathrm{APN}$ and for BA with or without $\beta \mathrm{APN}$. 


\section{CONTROL (nO BAPN)}

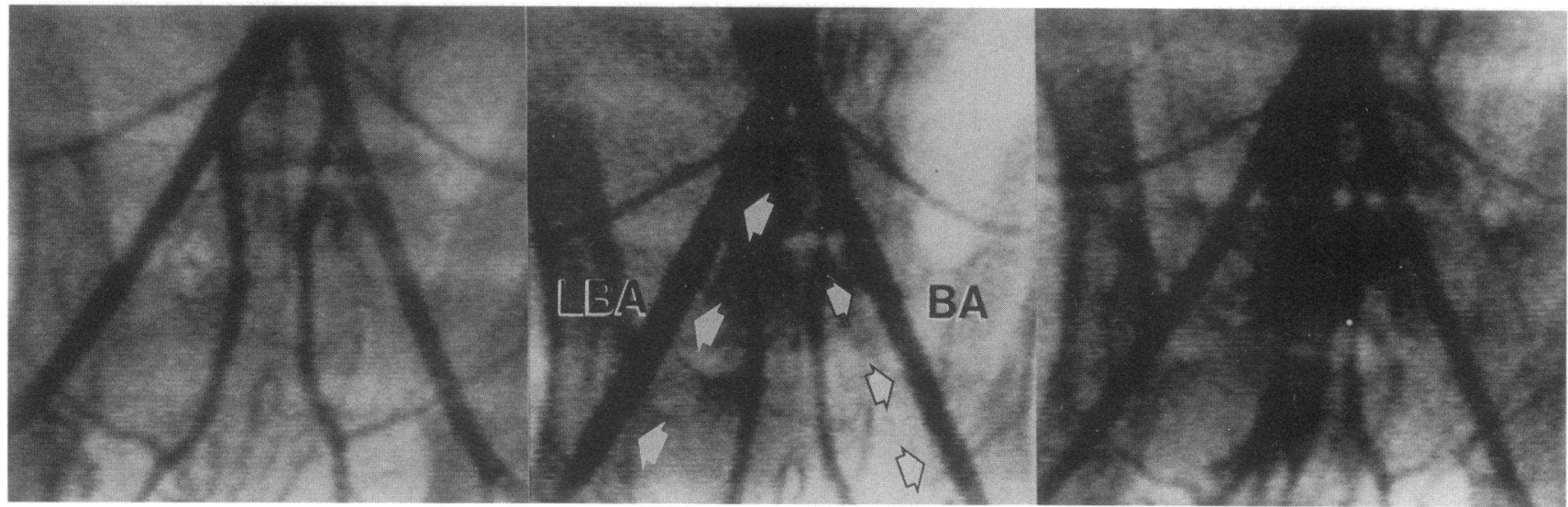

\section{BAPN}

PRE POST

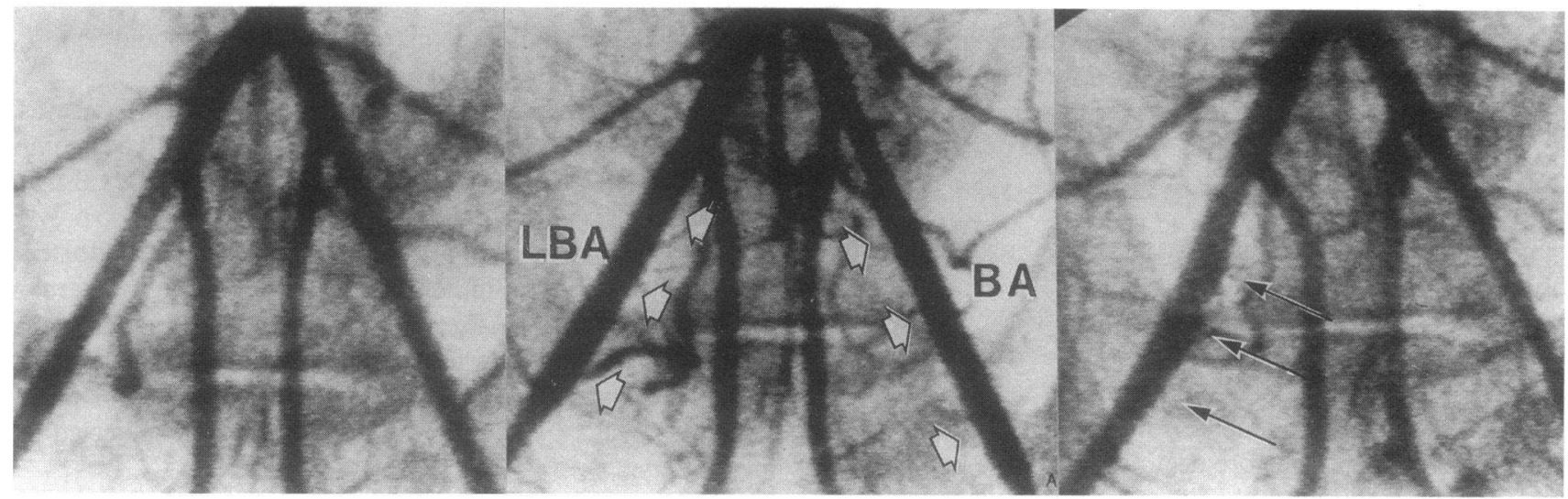

Figure 3. Angiograms from representative control (no $\beta A P N)$ and $\beta \mathrm{APN}$-treated rabbits before (PRE), acutely after (POST), and 1 mo after treatment of the external iliac arteries with ipsilateral BA and contralateral LBA. Large white arrows denote arterial segments subjected to angioplasty. Small black arrows indicate the arterial segment that had been subjected to laser/thermal exposure in the 1-month follow-up angiographic study.

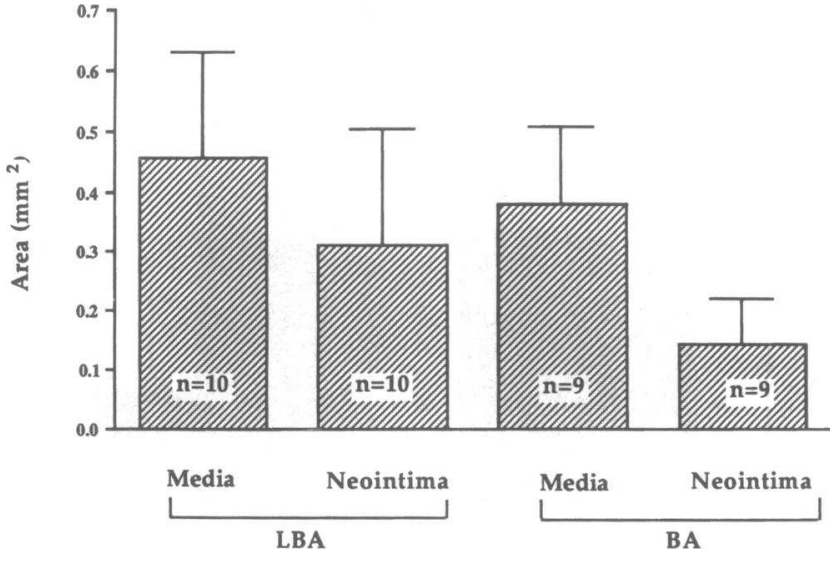

Figure 4. Medial and neiontimal areas by quantitative histology 1 mo after BA and LBA of the normal rabbit iliac artery. Note that neointimal areas are less than medial areas; the contribution of neointimal thickening to postangioplasty luminal diameter loss is minor (see text). Error bars represent \pm 1 SD. treated arteries 1 mo after angioplasty, DHLNL content was significantly increased to threefold that of control values; HLNL content also showed a slight, but significant, increase. In contrast, there were no significant differences in DHLNL or HLNL content of BA-treated arteries as compared with control values. $\beta A P N$ administration was associated with a significant decrease in DHLNL content relative to values in animals subjected to LBA without $\beta$ APN administration. However, values in this group were still significantly higher than control values. In contrast to DHLNL content in LBA-treated animals, HLNL content in this group was not affected by $\beta A P N$. Animals subjected to either BA alone or to BA plus $\beta A P N$ did not differ significantly from control animals with respect to any of the crosslink parameters.

DHLNL values in animals subjected to LBA 6 mo previously were less than half the values of those of the animal subjected to LBA 1 mo previously. However, DHLNL values in this group were still significantly elevated relative to agematched control animals, due in part to the normal decrease with aging of reducible cross-links. Both DHLNL and HLNL 

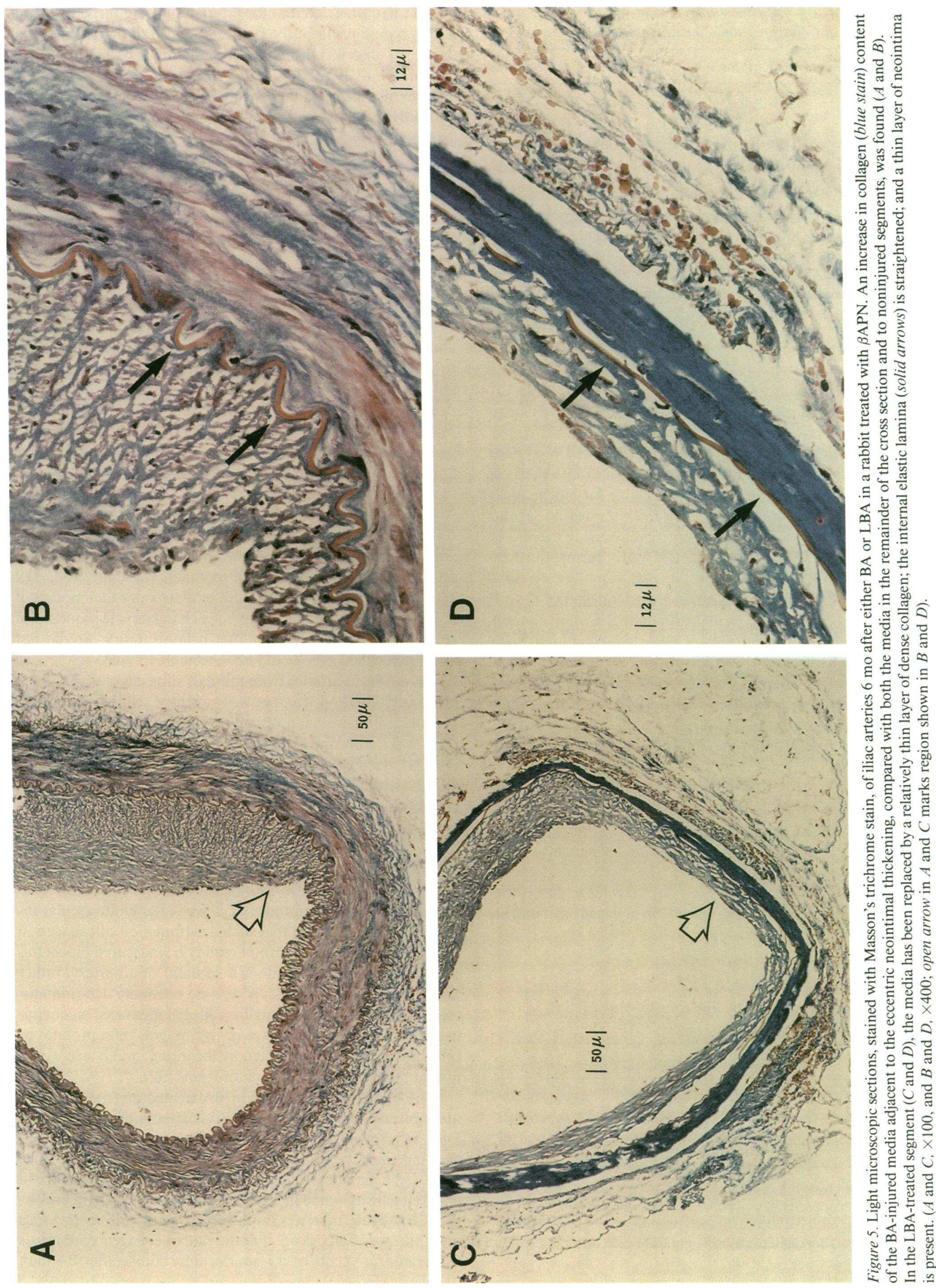
Table V. Comparison of Cross-link Content of Arterial Wall Collagen 1 mo after Angioplasty

\begin{tabular}{lccc}
\hline \multicolumn{1}{c}{ Group $(n)$} & DHLNL & HLNL & OHP \\
\hline Control (10) & $0.082 \pm 0.031^{*}$ & $0.037 \pm 0.008^{*}$ & $0.153 \pm 0.043$ \\
LBA (2) & $0.272 \pm 0.011^{\ddagger}$ & $0.060 \pm 0.028^{\ddagger}$ & $0.158 \pm 0.011$ \\
BA (2) & $0.041 \pm 0.013^{*}$ & $0.027 \pm 0.006^{*}$ & $0.138 \pm 0.003$ \\
LBA + BAPN (6) & $0.207 \pm 0.047^{\S}$ & $0.073 \pm 0.010^{\ddagger}$ & $0.167 \pm 0.039$ \\
BA + BAPN (6) & $0.066 \pm 0.033^{*}$ & $0.023 \pm 0.010^{*}$ & $0.178 \pm 0.036$
\end{tabular}

Comparison of cross-link content, expressed as moles of cross-link per moles of collagen, of arterial wall collagen $1 \mathrm{mo}$ after LBA or BA, with or without $\beta \mathrm{APN}$ administration for 1 mo postangioplasty.

In each column, values with different footnote symbols are significantly different from each other $(P<0.05$ by ANOVA).

values were significantly lower in the older controls (Table VI) as compared with the younger controls (Table V). Of particular interest was the observation that DHLNL values in BA-treated animals was higher $6 \mathrm{mo}$ after angioplasty than at $1 \mathrm{mo}$; these values were also significantly higher than control values. It should be noted that tissue was not available for biochemical analysis in the $\beta A P N$ group at the 6-mo time period, as the original experimental design was aimed at assessing the effects of $\beta \mathrm{APN}$ on biochemical parameters only during the first month.

OHP. OHP was readily detectable in arterial collagen. At 1 mo after angioplasty, there were no differences in OHP content of arterial collagen in either BA- or LBA-treated animals relative to control values. $\beta$ APN administration had no effect on OHP content 1 mo after angioplasty. However, 6 mo after angioplasty in the group without $\beta \mathrm{APN}$, OHP was significantly elevated relative to controls in both BA- and LBA-treated arteries. As noted above, tissue was not available for biochemical analysis in the $\beta$ APN group at the 6-mo time point.

\section{Discussion}

In this present study, we report on structural changes in arterial collagen after endovascular injury, and the effects of lathyrogen administration on such changes. To our knowledge, this is the first report of the effects of angioplasty on collagen structure at the molecular level.

Table VI. Comparison of Cross-link Content 6 mo after Angioplasty

\begin{tabular}{lccc}
\hline Group $(n)$ & DHLNL & HLNL & OHP \\
\hline Control (8) & $0.036 \pm 0.009^{*}$ & $0.017 \pm 0.005^{*}$ & $0.174 \pm 0.035^{*}$ \\
LBA (3) & $0.128 \pm 0.028^{\ddagger}$ & $0.045 \pm 0.007^{\ddagger}$ & $0.291 \pm 0.064^{\ddagger}$ \\
BA (3) & $0.100 \pm 0.017^{\ddagger}$ & $0.035 \pm 0.10^{\ddagger}$ & $0.251 \pm 0.074^{\ddagger}$ \\
\hline
\end{tabular}

Comparison of cross-link content, expressed as moles of crosslink per mole of collagen, of arterial wall collagen 6 mo after LBA or BA in rabbits not administered $\beta$ APN. In each column, values with different footnote symbols are significantly different from each other $(P<0.05$ by ANOVA).
We hypothesized that the arterial injury induced by angioplasty would result in the classic wound-healing response that has been observed in matrix in many other tissues and organ systems. Initially, there is biosynthesis of collagen characterized by increased content of the difunctional collagen crosslink DHLNL (i.e., an increase in moles of DHLNL/moles of collagen). Collagen content of DHLNL generally returns to normal over time; however, in many types of injury a delayed reponse will occur, characterized by increased collagen content of OHP, the maturational product of DHLNL. This structural abnormality is associated with alterations in the physicochemical properties of collagen, including increased insolubility and stiffness $(26,27)$, and is found in various fibrotic disorders characterized by increased deposition of collagen at the site of injury. Recent reviews have described this sterotypical pattern of structural change in collagen in skin, lung, tendon, bone, and cartilage in association with mechanical injury, exposure to various toxins, and certain congenital and acquired diseases $(11,26,28)$. In some injury models these may be only barely detectable, and occasionally either the early or late changes may not be apparent at all (29). A likely reason for such findings is that in some models there is insufficient synthesis of "new," abnormal collagen relative to the normal collagen already present in the given tissue or organ to permit detection of structural changes. Although it is possible to distinguish "new" collagen from "old" collagen in vivo by injecting radiolabeled amino acids at the time of injury, such experiments are technically very difficult and subject to much variability $(30,31)$. Thus, although our earlier studies of matrix in other models of injury and disease led us to predict a characteristic sequence of changes in vascular collagen after balloon injury, we did not know if such changes would be detectable in vivo.

However, in arteries from animals subjected to LBA 1 mo previously, DHLNL content was increased relative to controls as expected. There was no change in OHP at $1 \mathrm{mo}$, but by $6 \mathrm{mo}$ OHP was significantly increased relative to controls. Although DHLNL decreased significantly in LBA between 1 and 6 mo, DHLNL values were still higher than those of age-matched controls. These findings are consistent with much of our previous work (K. M. Reiser; 26, 28) on the response of matrix biosynthesis to injury and disease. Indeed, the observation that arterial collagen contains increased OHP 6 mo after balloon injury is consistent with histological evidence of excess accumulation of collagen in the arterial wall after endovascular injury. It should be noted that such accumulation of collagen in balloon-injured arteries cannot readily be measured biochemically because the injury site is a segment of an artery, rather than a whole organ. Thus, there is no adequate denominator term to which one can normalize collagen measured biochemically. Such parameters as wet or dry weight, total protein, or cell content may all be affected by the injury, and hence would not serve as reliable denominator terms (32).

The biochemical changes in BA-treated arteries were not identical to those observed in LBA-treated arteries. Although DHLNL showed no changes by 1 mo after angioplasty, significant increases were observed 6 mo after injury. OHP was also increased $6 \mathrm{mo}$ after injury. In view of the marked difference in collagen density noted histologically at follow-up between BAand LBA-treated arteries, both in this study and in previous ones, the possibility exists that the rate of collagen synthesis is slower and/or occurs over a shorter time course after BA than 
LBA injury, and the amount of newly synthesized abnormal collagen is insufficient to allow detection of increased levels of DHLNL since there is more normal collagen present relative to newly synthesize collagen. By analogy, we have observed that in models of lung injury the period during which increased DHLNL is detectable may vary depending on the agent used. For example, in lung injury induced by a single intratracheal instillation of bleomycin, DHLNL values are significantly elevated 1 wk after injury, but return to normal by 1 mo (31). In silica-induced injury, however, DHLNL elevations are readily detectable for several months after injury (33). Alternatively, laser/thermal exposure may denature collagen, thereby promoting its degradation by proteases to which it would ordinarily be resistant, so that only newly synthesized collagen is available for analysis, while in the BA-treated arteries a mixture of newly synthesized and normal collagen already present is available for analysis. This possibility is supported by recent evidence that laser exposure, at levels of thermal injury similar to the one used herein, does indeed structurally alter type I collagen by causing widespread denaturation (34). The crosslink data are consistent with both of these hypotheses, in that it appears that newly synthesized collagen containing excess DHLNL is not present in sufficient quantities to permit detection by 1 mo after BA-induced injury, whether because of slower synthesis than in LBA-induced injury, or because of the presence of "normal" collagen already present. However, the data clearly show that synthesis of such abnormal collagen does indeed occur after both types of angioplasty injury, as is evident by increased DHLNL and OHP at 6 mo.

The second major aim of our study was to investigate the effects of $\beta A P N$, a potent inhibitor of lysyl oxidase (12), on collagen structure after endovascular injury. We found that, for LBA/ $\beta A P N$-treated arteries, DHLNL was significantly decreased 1 mo after angioplasty compared with control LBA arteries, but was still higher than that for arteries not subjected to angioplasty. In contrast, $\beta$ APN had no significant effect on cross-links in arteries 1 mo after BA, but the DHLNL content of the control BA arteries was relatively low as discussed above. Interestingly, the effects of $\beta A P N$ on cross-links were mirrored by the angiographic data (Tables I-IV and Figs. 1-3). Only arteries subjected to LBA responded to $\beta$ APN with reduced luminal narrowing, irrespective of whether the 1-mo luminal diameter was compared with luminal diameter acutely before or after angioplasty; $\beta \mathrm{APN}$ had no significant effect on arteries subjected to BA.

What might be the mechanism of the differential response between the two angioplasty modalities? Given the markedly beneficial response of LBA to $\beta$ APN, it is likely that cross-linking of newly synthesized collagen is the predominant mechanism of the usual late loss in diameter of the rabbit iliac artery after this angioplasty modality. That this mechanism would be operative after LBA should not be surprising, in view of the initial inflammatory reaction and late prominent collagen deposition noted experimentally in the media histologically. Although $\beta A P N$ may inhibit elastin cross-linking $(35,36)$, there was no histologic evidence of increased elastin content at either 1 or 6 mo after LBA. However, $\beta$ APN may alter the cellular response to injury $(37,38)$, so that the potential additional effect of other mechanisms cannot be excluded. Considering the observation that the endogenous concentration of monoamine oxidase is relatively high in the rabbit, thereby poten- tially reducing the effectiveness of $\beta A P N$ in this species (39, 40 ), the response of LBA-treated arteries to this agent may be even more remarkable.

The principle mechanism(s) responsible for the late loss in diameter after BA of the normal rabbit iliac artery is less clear. Neointimal thickening $1 \mathrm{mo}$ after angioplasty was found to contribute to only a minor portion of the loss in diameter after either BA or LBA, and medial thickness was found to be similar to that reported by others for the normal rabbit femoral artery (41). Several groups of investigators have suggested that visco-elastic recoil frequently contributes to luminal compromise after conventional balloon angioplasty $(42,43)$. Such recoil appears to be reduced by thermal remodeling of connective tissue during LBA, and the straightening of the elastic lamellae found at 1 and 6 mo after laser exposure in this and prior studies $(18,44)$ is consistent with this notion. One would not expect that mechanical recoil after BA is amenable to pharmacologic inhibition. The lack of a significant response of BA treated arteries to a vasodilator at $1 \mathrm{mo}$ after angioplasty makes underlying vasoconstriction an unlikely mechanism for the late loss in luminal diameter. The possibility also exists that a stretch injury of preexisting mature connective tissue contributes to pathologic cross-linking of collagen and/or elastin. If so, thermal denaturation of structural proteins during LBA might inhibit the latter response, and lysyl oxidase inhibitors affect cross-linking only of newly synthesized collagen. Although the response of the injured luminal surface may differ between BA and LBA (45) in terms of platelet deposition, thrombogenicity, rate of reendothelialization, etc., the neointima appeared qualitatively and quantitatively similar between the two modalities, and the contribution of neointimal thickening to the late loss in diameter was minor.

The most important limitation of this study is that the pathogenesis of luminal compromise after angioplasty of atherosclerotic coronary artery lesions clinically is undoubtedly considerably more complex than that responsible for the late loss in diameter in this rabbit model. However, the magnitude of the absolute loss in luminal diameter noted experimentally herein and previously (18) is similar to that reported clinically after both BA (46) and elective LBA (47). More importantly, use of the model permits examination of the role of potentially important, alternative mechanisms of late luminal compromise independent of overt thrombus formation and prominent neointimal thickening. In addition, prominent intramural fibrosis may characterize some clinical angioplasty procedures other than LBA, e.g., conventional balloon angioplasty that is accompanied by extensive mechanical injury and stent implantation that may produce a foreign body tissue reaction (48). Additional studies would be required, of course, before extrapolation of the results of the use of $\beta A P N$ in conjunction with LBA to other angioplasty modalities.

In summary, our results suggest that the arterial wall is characterized by structural abnormalities of collagen after angioplasty injury, and that the type of angioplasty modality used is a critical variable in influencing matrix biosynthesis. $\beta A P N$ administration for 1 mo after LBA reduced luminal narrowing for up to $6 \mathrm{mo}$ after the procedure, thus demonstrating the potentially important role of collagen cross-linking and the feasibility of pharmacologic inhibition of the diameter loss. However, whether inhibition of collagen cross-linking would have utility for any angioplasty modality other than LBA is un- 
known. Regarding LBA-induced tissue responses, additional studies would have to be performed to determine an optimal regimen for modulation of matrix deposition, as agents are available for pharmacologic intervention at many stages of matrix biosynthesis. One can intervene at the transcriptional or translational level with nonspecific antimetabolites, at the stage of helical assembly (necessary for membrane transport) by administration of proline analogues, and finally, at the stage of fibril assembly in the extracellular matrix with lathyrogenic agents, which may have one of several mechanisms for interfering with fibril formation. Future studies will be aimed at elucidating the timing of events that occur in the biosynthesis of abnormal collagen after endovascular injury, thus potentially permitting the design of more precise regimens for modulating arterial response.

\section{Acknowledgments}

We are indebted to Jie Jang and Susan DeSautel for expert technical assistance, and to Barbara Fromm, M. A., for assistance in the statistical analysis.

This work was supported in part by National Institutes of Health grants HL-37349, HL-44683 (J. R. Spears), and RR-00169, AG-05324 (K. M. Reiser).

\section{References}

1. Califf, R. M., D. F. Fortin, D. J. Frid, W. R. Harlan III, E. M. Ohman, J. R. Bangtson, C. L. Nelson, J. E. Tcheng, D. B. Mark, and R. S. Stack. Restenosis after coronary angioplasty: an overview. 1991. J. Am. Coll. Cardiol. 17:2B-13B.

2. Ross, R. 1986. The pathogenesis of atherosclerosis-an update. $N$. Engl. J. Med. 314:488-500.

3. Harker, L. A. 1987. Role of platelets and thrombosis in mechanisms of acute occlusion and restenosis after angioplasty. Am. J. Cardiol. 60:20B-28B.

4. Essed, C. E., M. van den Brand, and A. E. Becker. 1983. Transluminal coronary angioplasty and early restenosis: fibrocellular occlusion after wall laceration. Br. Heart J. 49:393-396.

5. Austin, G. E., N. B. Ratliff, F. Hollman, S. Tabei, and D. R. Phillips. 1985. Intimal proliferation of smooth muscle cells as an explanation for recurrent coronay artery stenosis after percutaneous transluminal angioplasty. J. Am. Coll. Cardiol. 6:369-375.

6. Nobuyoshi, M., T. Kimura, H. Ohishi, H. Horiuchi, H. Nosaka, N. Hamasaki, H. Yokoi, and K. Kim. 1991. Restenosis after percutaneous transluminal coronary angioplasty: pathologic observations in 20 patients. J. Am. Coll. Cardiol. 17:433-439.

7. Pickering, J. G., L. Weir, K. Rosenfield, J. Stetz, J. Jekanowski, and J. M. Isner. 1992. Smooth muscle cell outgrowth from human atherosclerotic plaque: implications for the assessment of lesion biology. J. Am. Coll. Cardiol. 15:14301439.

8. Clowes, A. W., M. A. Reidy, M. M. Clowes. 1983. Mechanism of stenosis after arterial injury. Lab. Invest. 49:208-215.

9. Schwartz, R. S., K. C. Huber, J. G. Murphy, W. D. Edwards, A. R. Camrud, R. E. Vliestra, and D. R. Holmes. 1992. Restenosis and the proportional neointimal response to coronary artery injury: results in a porcine model. J. Am. Coll. Cardiol. 19:267-274.

10. Strauss, B. H., V. A. Umans, R.-J. van Suylen, P. J. de Feyter, J. Marco, G. C. Robertson, J. Renkin, G. Heyndrickx, V. D. Vuzevski, F. T. Bosman, and P. W. Serruys. 1992. Directional atherectomy for treatment of restenosis within coronary stents: clinical, angiographic and histologic results. J. Am. Coll. Cardiol. 20:1465-1473.

11. Reiser, K. M., R. McCormick, and R. B. Rucker. 1992. Enzymatic and nonenzymatic cross-linking of collagen and elastin. FASEB (Fed. Am. Soc. Exp. Biol.) J. 6:2439-2449.
12. Tang, S.-S. , P. C. Trackman, and H. M. Kagan. 1983. Reaction of aortic lysyl oxidase with beta-aminopropionitrile. J. Biol. Chem. 258:4331-4338.

13. Levene, C. I. 1973. Lathyrism. In Molecular Pathology of Connective Tissues. R. Perez-Tamayo and M. Rojkind, editors. Marcel Dekker, Inc., New York. 175-228.

14. Peacock, E. E., Jr. 1981. Control of wound healing and scar formation in surgical patients. Arch. Surg. 116:1325-1329.

15. Riley, D. J., J. S. Kerr, R. A. Berg, B. D. Ianni, G. G. Pietra, N. H. Edelman, and D. J. Prockop DJ. 1982. Beta-aminopropionitrile prevents bleomycin-induced pulmonary fibrosis in the hamster. Am. Rev. Respir. Dis. 125:6773.

16. Moorehead, L. C., J. Smith, R. Stewart, and R. Kimbrough. 1987. Effects of beta-aminopropionitrile after glaucoma filtration surgery: pilot human trial. Ann. Ophthalmol. 19:223-225.

17. Lata, A., C. Gowri, S. C. Dhar, J. D. Gibeault, and M. Chvapil. 1988. Effect of topically applied beta-aminopropionitrile on granuloma tissue biochemistry. Proc. Soc. Exp. Biol. Med. 187:442-447.

18. Jenkins, R. D., I. N. Sinclair, B. M. Leonard, T. Sandor, F. J. Schoen, and J. R. Spears. 1989. Laser balloon angioplasty versus balloon angioplasty in normal rabbit iliac arteries. Lasers Surg. Med. 9:237-247.

19. Cheong, W.-F., J. R. Spears, and A. J. Welch. 1991. Laser balloon angioplasty. Crit. Rev. Biomed. Eng. 19:113-146.

20. Spears, J. R., T. Sandor, A. V. Als, M. Malagold, J. E. Markis, W. Grossman, J. R. Serur, and S. Paulin. 1983. Computerized image analysis for quantitative measurement of vessel diameter from cineangiograms. Circulation. 68:453461

21. Sandor, T., and J. R. Spears. 1985. Statistical consideration on the precision of assessing blood vessel diameter in cine coronary angiography. Comput. Biomed. Res. 18:531-543.

22. Sandor, T., A. D'Adamo, W. B. Hanlon, and J. R. Spears. 1987. High precision quantitative angiography. IEEE (Inst. Electr. Electron. Eng.) Trans. Med. Imag. MI-6:258-265.

23. Reiser, K. M., and J. A. Last. 1983. Analysis of collagen composition and biosynthesis by high performance liquid chromatography. Liquid Chromatography 1:498-502.

24. Buckingham, B., and K. M. Reiser. 1990. Relationship between the content of lysyl oxidase-dependent cross-links in skin collagen, nonenzymatic glycosylation, and long-term complications in type I diabetes mellitus. J. Clin. Invest. 86:1046-1054.

25. Woessner, J. F. 1961. Determination of hydroxyproline in tissue and protein samples containing small proportions of this imino acid. Arch. Biochem. Biophys. 93:440-447.

26. Reiser, K. M., and J. A. Last. 1986. Collagen crosslinking in lungs of rats with experimental silicosis. Collagen Relat. Res. 6:313-323.

27. Eyre, D. R., T. J. Koob, and K. P. Van Ness. 1984. Quantitation of hydroxypryidinium crosslinks in collagen by high-performance liquid chromatography. Anal. Biochem. 137:380-388.

28. Last, J. A., L. G. Armstrong, and K. M. Reiser. 1990. Biosynthesis of collagen crosslinks. Int. J. Biochem. 22:559-564.

29. Haschek, W. M., K. M. Reiser, A. J. Klein-Szanto, J. P. Kehrer, L. H Smith, J. A. Last, and H. P. Witschi. 1983. Potentiation of butylated hydroxytoluene-induced acute lung damage by oxygen. Cellular kinetics and collagen metabolism. Am. Rev. Respir. Dis. 127:28-34.

30. Last, J. A., P. Summers, and K. M. Reiser. 1989. Biosynthesis of collagen crosslinks. II. In vivo labelling and stability of lung collagen in rats. Biochim. Biophys. Acta. 990:182-189.

31. Last, J. A., and K. M. Reiser. 1989. Biosynthesis of collagen crosslinks. III. In vivo labeling and stability of lung collagen in rats with bleomycin-induced pulmonary fibrosis. Am. J. Respir. Cell Mol. Biol. 1:111-117.

32. Greenberg, D. B., K. M. Reiser, and J. A. Last. 1978. Correlation of biochemical and morphologic manifestations of acute pulmonary fibrosis in rats administered paraquat. Chest. 74:421-425.

33. Reiser, K. M., and J. A. Last. 1986. Collagen crosslinking in lungs of rats with experimental silicosis. Collagen Relat. Res. 6:313-323.

34. Bass, L. S., N. Moazami, J. Pocsidio, M. C. Oz, P. LoGerfo, and M. R. Treat. 1992. Changes in type I collagen following laser welding. Lasers Surg. Med. 12:500-505.

35. Eyre, D. R., M. A. Paz, and P. M. Gallop. 1984. Cross-linking in collagen and elastin. Annu. Rev. Biochem. 53:717-748.

36. Capdeville, M., M. Coutard, and M. J. Osborne-Pellegrin. Spontaneous rupture of the internal elastic lamina in the rat: the manifestation of a genetically determined factor which may be linked to vascular fragility. 1989. Blood Vessels. 26:197-212

37. Hurley, J. V., E. Storey, and K. N. Ham. 1958. The effect of amino-acetonitrile and cortisone on the healing of turpentine-induced abscesses in the rat. Br. J. Exp. Pathol. 39:119-127. 
38. Nelson, J. M., R. F. Diegelmann, and I. K. Cohen. 1988. Effect of betaaminopropionitrile and ascorbate on fibroblast migration. Proc. Soc. Exp. Biol. Med. 188:346-352.

39. Fleisher, J. H., D. Speer, K. Brendel, and M. Chvapil. 1979. Effects of pargyline on the metabolism of beta-aminopropionitrile ( $\beta \mathrm{APN}$ ) by rabbits. Toxicol. Appl. Pharmacol. 40:61-69.

40. Moorehead, L. C. 1983. Effects of beta-aminopropionitrile after posterior penetrating injury in the rabbit. Am. J. Ophthalmol. 95:97-109.

41. Crawford, D. W., L. H. Back, and M. A. Cole. 1980. In vivo oxygen transport to the normal rabbit femoral arterial wall. J. Clin. Invest. 65:14981508.

42. Spears, J. R. 1987. Percutaneous transluminal coronary angioplasty restenosis: potential prevention with laser balloon angioplasty. Am. J. Cardiol. 60:61B-64B.

43. Waller, B. F., and C. A. Pinkerton. 1990. "Cutters, scoopers, shavers, and scrapers": the importance of atherectomy devices and clinical relevance of tissue removed. J. Am. Coll. Cardiol. 15:426-428.

44. Spears, J. R. 1990. Laser balloon angioplasty: experimental in vivo and in vitro studies. In Lasers in Cardiovascular Medicine and Surgery: Fundamentals and Techniques. G. S. Abela, editor. Kluwer Academic Publishers, Norwell, MA 167-188.

45. Spears, J. R., S. K. Kundu, and L. P. McMath. 1991. Laser balloon angioplasty: potential for reduction of the thrombogenicity of the injured arterial wall and for local application of bioprotective materials. J. Am. Coll. Cardiol. 17:179B-188B.

46. Nobuyoshi, M., T. Kimura, H. Nosaka, S. Mioka, K Ueno, H. Yokoi, N. Hamasaki, H. Horiuchi, H. Ohishi. 1988. Restenosis after successful percutaneous transluminal coronary angioplasty: serial angiographic follow-up of 229 patients. J. Am. Coll. Cardiol. 12:616-623.

47. Spears, J. R. 1992. Improved luminal dimensions and local pharmacologic therapy with laser balloon angioplasty for potential mitigation of angioplasty restenosis. In Restenosis after Intervention with New Mechanical Devices P. W. Serruys, B. H. Strauss, and S. B. King III, editors. Kluwer Academic Publishers, Dordrecht. 347-358.

48. Schwartz, R. S., J. G. Murphy, W. D. Edwards, A. R. Camrud, R. E. Vlietstra, D. R. Holmes. 1990. Restenosis after balloon angioplasty. A practical proliferative model in porcine coronary arteries. Circulation. 82:2190-2200. 